\title{
Comparative effectiveness of adjuvant chemoradiotherapy after gastrectomy among older patients with gastric adenocarcinoma: a SEER-Medicare study
}

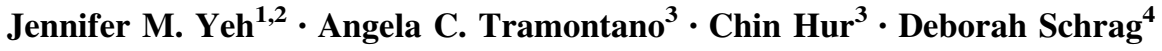

Received: 22 December 2016/Accepted: 14 January 2017/Published online: 15 February 2017

(c) The International Gastric Cancer Association and The Japanese Gastric Cancer Association 2017

\begin{abstract}
Background Since the INT-0116 trial reported a survival advantage, postoperative chemoradiotherapy (CRT) has been a care standard for US patients in whom gastric adenocarcinoma has been diagnosed. We sought to estimate the association between treatment and survival among the older US Medicare population.

Methods This is a retrospective cohort study of Medicare beneficiaries aged 65-79 years with stage IB-III gastric adenocarcinoma diagnosed between 2002 and 2009 in a Surveillance, Epidemiology, and End Results region. Patients were categorized on the basis of treatment: (1) gastrectomy only and (2) gastrectomy plus adjuvant CRT. We examined factors associated with receipt of adjuvant CRT, including stage at diagnosis, comorbidity, and tumor subtype. Overall survival was measured from 90 days after gastrectomy until death or the censoring date of December 31, 2010.
\end{abstract}

Electronic supplementary material The online version of this article (doi:10.1007/s10120-017-0693-x) contains supplementary material, which is available to authorized users.

Jennifer M. Yeh

jennifer.yeh@childrens.harvard.edu

1 Division of General Pediatrics, Boston Children's Hospital, Harvard Medical School, 300 Longwood Avenue, Boston, MA 02115, USA

2 Center for Health Decision Science, Harvard T.H. Chan School of Public Health, Boston, MA, USA

3 Institute for Technology Assessment, Massachusetts General Hospital, Boston, MA, USA

4 Dana-Farber Cancer Institute, Harvard Medical School, Boston, MA, USA
Results Of the 1519 patients who underwent gastrectomy, $41.7 \%$ received adjuvant CRT. Factors associated with adjuvant CRT included age younger than 75 years at cancer diagnosis and stage II or stage III cancer. The median overall survival from the time of gastrectomy was 25.1 months (interquartile range 43.7 months) for gastrectomy only and 26.9 months (interquartile range 40.9 months) for adjuvant CRT. Multivariable and propensity-score-stratified models demonstrated a survival benefit associated with adjuvant CRT [hazard ratio (HR) 0.58 ; 95\% confidence interval (CI) $0.50-0.67$ ], although the magnitude was greater for stage II tumors (HR 0.50; 95\% CI 0.39-0.61) and stage III tumors (HR 0.58; 95\% CI 0.45-0.73) than for stage IB tumors (HR 1.02; 95\% CI 0.71-1.45).

Conclusions Adjuvant CRT, in conjunction with gastrectomy, was associated with a survival benefit among older patients with stage II or stage III tumors.

Keywords Gastric cancer - Adjuvant chemoradiotherapy · SEER-Medicare · Comparative effectiveness research

\section{Introduction}

Gastric cancer is the third leading cause of cancer-related death worldwide, responsible for nearly $9 \%$ of all cancer deaths [1]. Each year, gastric cancer is diagnosed in 20,000 individuals in the USA alone [2]. Although surgical resection of early-stage disease is associated with survival rates of more than $90 \%$, outcomes are less favorable for more advanced disease [3]. New adjuvant therapies are the focus of ongoing efforts to improve survival outcomes for patients in whom gastric adenocarcinoma has been diagnosed. 
Randomized clinical trials, including the Southwest Oncology Group (SWOG) 9008/Intergroup 0116 (INT0116) $[4,5]$ and Medical Research Council Adjuvant Gastric Infusional Chemotherapy (MAGIC) [6] studies, have led to the addition of postoperative chemoradiotherapy (CRT) or perioperative chemotherapy to gastrectomy as standard therapy in the USA [7-9]. However, the utilization rates for these regimens are low (less than $20 \%$ and less than $2 \%$ respectively) [10], with uncertain effectiveness among the general population.

Studies based on Surveillance, Epidemiology, and End Results (SEER) data report a survival benefit associated with adjuvant radiotherapy following gastrectomy [11-15]. However, because the SEER Program does not include detailed chemotherapy data, the benefit of CRT remains unclear. Debate further continues on whether the addition of adjuvant CRT provides an incremental treatment benefit or only compensates for inadequate surgical resection by decreasing locoregional recurrences [16-18]. As more than $60 \%$ of gastric cancer patients are 65 years or older when gastric cancer is diagnosed, the effectiveness of adjuvant therapy among older individuals is of particular clinical importance [2].

Providing patient information on chemotherapy treatment, linked SEER-Medicare data can provide more informative estimates of the survival benefit associated with CRT among older individuals in whom gastric cancer has been diagnosed. Previous studies using SEER-Medicare data collected before the adoption of CRT as standard care in 2002 report conflicting results on the survival benefit $[19,20]$. As such, we sought to provide updated estimates using SEER-Medicare data on patients treated since adjuvant CRT became standard care in 2002.

\section{Materials and methods}

\section{Data source}

We used the SEER-Medicare data to estimate the survival benefit associated with adjuvant CRT among individuals in whom gastric adenocarcinoma had been diagnosed and treated with gastrectomy. The database results from the linkage of two large population-based data sources: SEER cancer registries data and Medicare enrollment and claims files [21]. The SEER Program collects and publishes cancer incidence, prevalence, and survival data from populationbased cancer registries covering approximately $26 \%$ of the US population [22]. SEER includes data on patient demographics, cancer site, histologic type, stage, and grade, and dates of diagnosis and death [21, 23]. Medicare includes data on Part A and Part B claims for covered health care services, including hospital, physician, outpatient, home health, and hospice bills [21]. SEER data for patients with diagnoses between January 1, 2002 and December 31, 2009 were matched to Medicare claims data from January 1, 2001 (or July 1, 2000 for 6 months before diagnosis) through December 31, 2010.

\section{Study sample}

The retrospective study cohort included patients aged 65-79 years with nonmetastatic, pathologically confirmed, stage IB-III gastric adenocarcinoma as their primary diagnosis between January 1, 2002 and December 31, 2009 who underwent gastrectomy within 6 months of diagnosis (Fig. 1). We excluded patients who were (1) not continuously eligible for Medicare Part A and Part B for the 12-month period between 6 months before cancer diagnosis and 6 months after diagnosis, (2) enrolled in a health maintenance organization (HMO) at any time during the period, (3) received any neoadjuvant therapy (e.g. chemotherapy and/or radiation therapy before gastrectomy), or (4) died within 90 days of gastrectomy (based on the landmark analysis [24], which determined that more than $80 \%$ of patients received adjuvant CRT within 90 days of gastrectomy, to minimize immortal time bias). We excluded patients aged 80 years or older as there is no established benefit from adjuvant CRT in this age group, and therefore it is not considered standard care for this subpopulation. Staging information was based on the American Joint Committee on Cancer (AJCC) cancer staging manual (sixth edition) [25]. Patients in whom the AJCC stage could not be determined were excluded.

\section{Adjuvant CRT}

Variables were created to indicate gastrectomy within 6 months of diagnosis and receipt of chemotherapy and radiation therapy within 3 months after gastrectomy. Detailed information on the codes used to capture Medicare claims for gastrectomy, radiation therapy, and chemotherapy can be found in Table S1. Patients who had at least one Medicare claim with a gastrectomy code were coded as having had gastrectomy. Among these patients, we identified those who received chemotherapy or radiation therapy (or both) on the basis of Medicare claims. If the patient had at least one claim with a chemotherapy code from any Medicare file [Medicare Provider Analysis and Review (MEDPAR) (inpatient), Carrier Claims, Outpatient Claims, Home Health Agency, Hospice, and Durable Medical Equipment] and this claim was within 3 months of gastrectomy, the patient was coded as having had chemotherapy. Because Medicare Part D was not available for our entire study period, we included only capecitabine for oral chemotherapies. Patients receiving radiation 


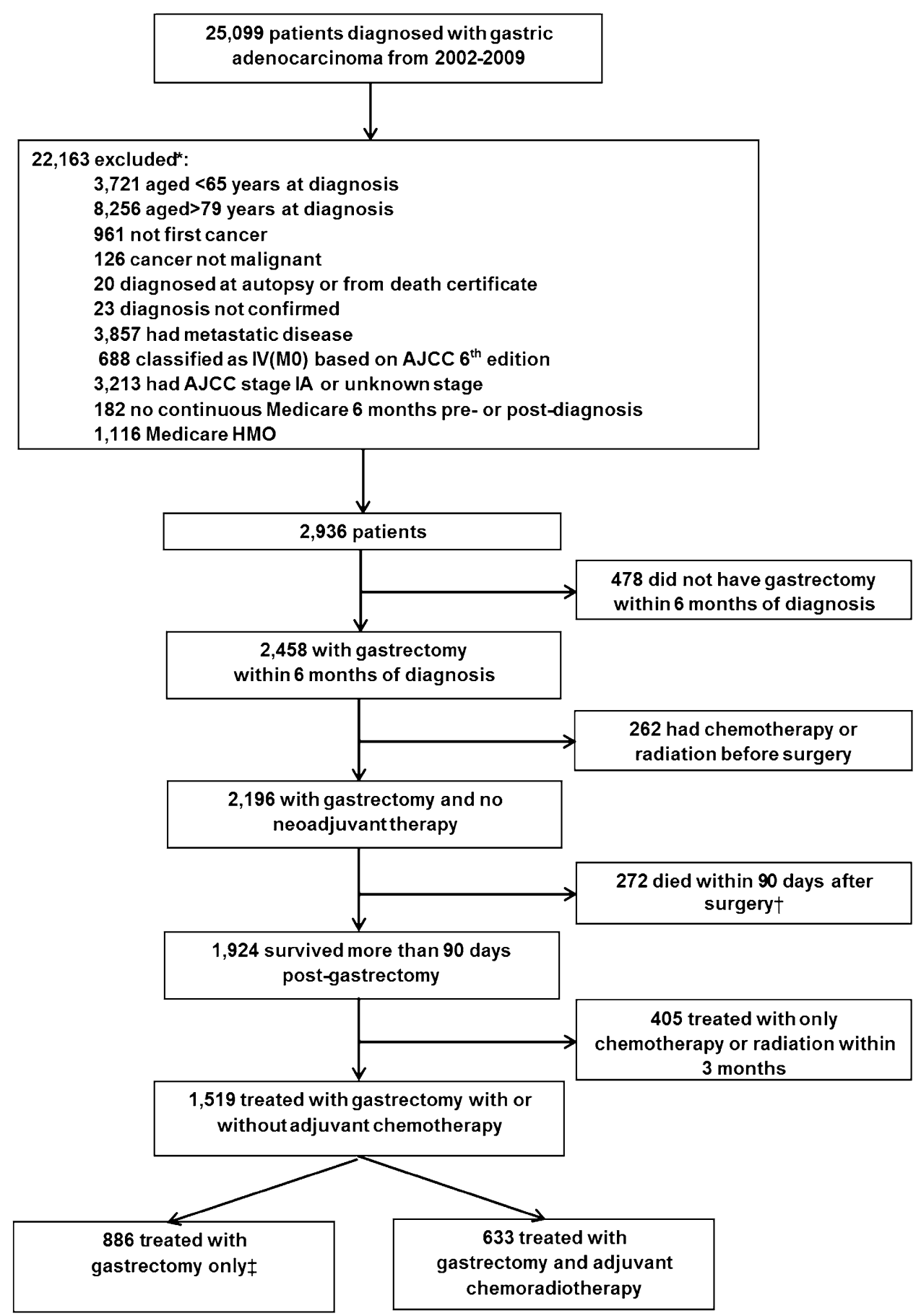

Fig. 1 Study cohort. Asterisk exclusions were sequential, dagger based on landmark analysis that determined more than $80 \%$ of individuals received adjuvant chemoradiotherapy within 90 days of gastrectomy, double dagger no adjuvant therapy within 3 months after gastrectomy, AJCC American Joint Committee on Cancer, HMO health maintenance organization

therapy were similarly identified by radiation-specific codes. To ensure complete information about radiation therapy, both SEER and Medicare variables were used to code for radiation therapy. Patients who had at least one radiation therapy claim in Medicare within 6 months of diagnosis were coded as having had adjuvant radiation therapy.

\section{Survival outcomes}

The primary outcome was all-cause death, defined as the number of survival months from 90 days after gastrectomy until death or December 31, 2010, whichever came first. The secondary outcome was cause-specific death. The date of death and the date of gastrectomy were identified from 
Medicare records. Patients treated with gastrectomy only and gastrectomy plus adjuvant CRT were defined as the control and treatment groups respectively. We censored observations for patients who were alive at the end of the follow-up. For the secondary analysis, cancer-specific death was coded by the SEER cause-specific death classification in the Patient Entitlement and Diagnosis Summary File (PEDSF).

\section{Baseline characteristics}

Demographic and clinical data included age, sex, race/ ethnicity, median income (census tract quintile), college education (census tract quintile), SEER geographic region, birth place, comorbidity, year of diagnosis, AJCC stage, tumor location, tumor histologic type, and number of lymph nodes resected (Table 1). Race/ethnicity was categorized as non-Hispanic white, non-Hispanic black, nonHispanic Asian, and Hispanic. Race in SEER is based on patients' medical records and registration information, while Hispanic ethnicity is determined through a Hispanicsurname algorithm. Tumor histologic type was categorized as intestinal, diffuse, and other tumors defined on criteria proposed by Lauren [26] and used by Henson et al. [27] and $\mathrm{Wu}$ et al. [28]. The number of lymph nodes resected was used as a proxy for determination of extent of lymphadenectomy (less than 15, 15-25, and more than 25). The burden associated with comorbidities was estimated by application of the modified Deyo adaptation [29-31] of the Charlson comorbidity index [32] to Medicare inpatient, outpatient, and physician claims during the 6-month period before cancer diagnosis. We classified the comorbidity into three groups with scores of 0,1 , and $2+$.

\section{Statistical analysis}

We used a chi square test to evaluate differences in the distribution of baseline characteristics between the treatment group and the control group. To estimate median survival, we used the Kaplan-Meier survival method and tests for crude differences among the groups using a logrank test.

To examine whether the adjuvant therapy improved overall survival in patients with gastric adenocarcinoma who underwent gastrectomy, we fitted two sets of Cox proportional hazards models for all-cause death. The first set of models estimated the overall relative hazard ratio between patients who underwent gastrectomy and received adjuvant CRT versus those treated with gastrectomy only. The second set of models estimated the stage-specific relative hazard ratios from the interaction between treatment effect and cancer stage at diagnosis (stages IB, II, and III).
For each set, we fitted a series of models. The first model estimated the unadjusted relative hazard ratio comparing gastrectomy plus adjuvant CRT with gastrectomy only. The second model adjusted this relative hazard ratio for all demographic and clinical characteristics listed in Table 2. As the treatment effect estimates are likely confounded by factors related to treatment selection, the third model accounted for measurable confounders between the groups using propensity score analysis [33]. This entailed use of multivariable logistic regression to estimate the likelihood of adjuvant therapy among patients who underwent gastrectomy based on confounding covariates, including age, sex, race/ethnicity, median income, college education, geographic region, modified Charlson comorbidiy scores, year of diagnosis, AJCC stage, tumor location, tumor histologic type, and number of lymph nodes resected [34, 35]. Each patient received a propensity score based on his/her predicted probability of receiving adjuvant therapy. Patients who had scores lower than the higher of the two minima or higher than the lower of the two maxima were excluded to prevent unreasonable extrapolation. Using the estimated propensity scores, we adjusted the data for group differences in four ways: (1) propensity score stratification, in which the propensity scores were divided into five strata and stratum-specific hazard ratios calculated with Cox proportional hazards models were combined for an overall hazard ratio [33, 36, 37]; (2) propensity score 1:1 matching, using an algorithm with a caliper of 0.02 [38] to pair adjuvant CRT and gastrectomy-only patients similar in terms of their measurable characteristics; (3) regression adjustment (i.e. inclusion of propensity score as a linear predictor in the model); and (4) creation of stabilization weights defined as the inverse probability of treatment weighting [39, 40]. We present adjusted relative hazard ratios and 95\% confidence intervals (CIs). An adjusted hazard ratio less than 1.00 indicated longer survival time among patients who underwent gastrectomy and received adjuvant CRT compared with those who underwent gastrectomy only, and an adjusted hazard ratio greater than 1.00 indicated shorter survival time.

To determine whether survival benefits differ by select patient and tumor characteristic, we performed subgroups analyses by cancer diagnosis age and tumor location. Sensitivity analyses evaluated the potential impact of defining adjuvant therapy on the basis of 2 or 4 months (versus 3 months in our base case analysis) from the date of gastrectomy and alternative definitions of adjuvant therapy that included any adjuvant chemotherapy (regardless of receipt of radiation therapy), only adjuvant chemotherapy, and only adjuvant radiation therapy.

All analyses were conducted with SAS, version 9.3 (SAS Institute, Cary, NC, USA). Statistical significance was set at $p<0.05$, and all tests were two-tailed. 
Table 1 Characteristics of older patients with gastric adenocarcinoma treated with gastrectomy for two treatment groups

\begin{tabular}{|c|c|c|c|}
\hline Characteristics & Gastrectomy only $(n=886)$ & $\begin{array}{l}\text { Gastrectomy plus adjuvant } \\
\text { CRT }(n=633)\end{array}$ & $p^{\mathrm{b}}$ \\
\hline \multicolumn{4}{|l|}{ Age at diagnosis (years) } \\
\hline $65-69$ & $201(22.7 \%)$ & $227(35.9 \%)$ & \multirow[t]{3}{*}{$<0.000$} \\
\hline $70-74$ & $289(32.6 \%)$ & $251(39.7 \%)$ & \\
\hline $75-79$ & $396(44.7 \%)$ & $155(24.5 \%)$ & \\
\hline \multicolumn{4}{|l|}{ Sex } \\
\hline Male & $545(61.5 \%)$ & $398(62.9 \%)$ & \multirow[t]{2}{*}{0.59} \\
\hline Female & $341(38.5 \%)$ & $235(37.1 \%)$ & \\
\hline \multicolumn{4}{|l|}{ Race/ethnicity } \\
\hline Non-Hispanic white & $488(55.1 \%)$ & $356(56.2 \%)$ & \multirow[t]{4}{*}{0.82} \\
\hline Non-Hispanic black & $137(15.5 \%)$ & $88(13.9 \%)$ & \\
\hline Non-Hispanic Asian/other & $148(16.7 \%)$ & $111(17.5 \%)$ & \\
\hline Hispanic & $113(12.8 \%)$ & $78(13.3 \%)$ & \\
\hline \multicolumn{4}{|l|}{ Median income } \\
\hline 0 (lowest) & $174(19.6 \%)$ & $93(14.7 \%)$ & \multirow[t]{5}{*}{0.0002} \\
\hline 1 & $170(19.2 \%)$ & $102(16.1 \%)$ & \\
\hline 2 & $185(20.9 \%)$ & $120(19.0 \%)$ & \\
\hline 3 & $187(21.1 \%)$ & $138(21.8 \%)$ & \\
\hline 4 (highest) & $170(19.2 \%)$ & $180(28.4 \%)$ & \\
\hline \multicolumn{4}{|l|}{ College educated } \\
\hline 0 (lowest) & $198(22.4 \%)$ & $119(18.8 \%)$ & \multirow[t]{5}{*}{0.0005} \\
\hline 1 & $187(21.1 \%)$ & $106(16.8 \%)$ & \\
\hline 2 & $183(20.7 \%)$ & $116(18.3 \%)$ & \\
\hline 3 & $175(19.8 \%)$ & $139(22.0 \%)$ & \\
\hline 4 (highest) & $143(16.1 \%)$ & $153(24.2 \%)$ & \\
\hline \multicolumn{4}{|l|}{ SEER region } \\
\hline Northeast & $178(20.1 \%)$ & $136(21.5 \%)$ & \multirow[t]{4}{*}{0.71} \\
\hline South & $193(21.8 \%)$ & $148(23.4 \%)$ & \\
\hline Midwest & $81(9.1 \%)$ & $53(8.4 \%)$ & \\
\hline West/Hawaii & $434(49.0 \%)$ & $296(46.8 \%)$ & \\
\hline \multicolumn{4}{|l|}{ Birthplace } \\
\hline USA and US territories & $451(50.9 \%)$ & $303(47.9 \%)$ & \multirow[t]{3}{*}{0.43} \\
\hline Foreign & $169(19.1 \%)$ & $135(21.3 \%)$ & \\
\hline Unknown & $266(30.0 \%)$ & $195(30.8 \%)$ & \\
\hline \multicolumn{4}{|l|}{ AJCC stage } \\
\hline $\mathrm{IB}$ & $401(45.3 \%)$ & $101(16.0 \%)$ & \multirow[t]{3}{*}{$<0.0001$} \\
\hline II & $309(34.9 \%)$ & $300(47.4 \%)$ & \\
\hline III & $176(19.9 \%)$ & $232(36.7 \%)$ & \\
\hline \multicolumn{4}{|l|}{ Charlson comorbidity score ${ }^{a}$} \\
\hline 0 & $424(47.9 \%)$ & $334(52.8 \%)$ & \multirow[t]{3}{*}{0.07} \\
\hline 1 & $253(28.6 \%)$ & $179(28.3 \%)$ & \\
\hline $2+$ & $209(23.6 \%)$ & $120(19.0 \%)$ & \\
\hline \multicolumn{4}{|l|}{ Tumor location } \\
\hline Cardia/overlapping & $330(37.3 \%)$ & $219(34.6 \%)$ & \multirow[t]{2}{*}{0.29} \\
\hline No-cardia & $556(62.8 \%)$ & $414(65.4 \%)$ & \\
\hline \multicolumn{4}{|l|}{ Lauren classification } \\
\hline Intestinal & $640(72.2 \%)$ & $431(68.1 \%)$ & \multirow[t]{3}{*}{0.02} \\
\hline Diffuse & $164(18.5 \%)$ & $153(24.2 \%)$ & \\
\hline Other & $82(9.3 \%)$ & $49(7.7 \%)$ & \\
\hline
\end{tabular}


Table 1 continued

\begin{tabular}{lll}
\hline Characteristics & Gastrectomy only $(n=886)$ & $\begin{array}{l}\text { Gastrectomy plus adjuvant } \\
\text { CRT }(n=633)\end{array}$ \\
\hline $\begin{array}{l}\text { Year of diagnosis } \\
2002-2004\end{array}$ & $437(49.3 \%)$ & $292(46.1 \%)$ \\
$2005-2007$ & $313(35.3 \%)$ & $210(33.2 \%)$ \\
$2008-2009$ & $136(15.4 \%)$ & $131(20.7 \%)$ \\
Lymph nodes examined & & \\
$<15$ & $555(62.6 \%)$ & $344(54.3 \%)$ \\
$15-25$ & $212(23.9 \%)$ & $179(28.3 \%)$ \\
$>25$ & $104(11.7 \%)$ & $95(15.0 \%)$ \\
Unknown & $15(1.7 \%)$ & $15(2.4 \%)$ \\
\hline
\end{tabular}

The percentages are rounded to the nearest tenth of a percent.

AJCC American Joint Committee on Cancer, CRT chemoradiotherapy, SEER Surveillance, Epidemiology, and End Results

${ }^{\text {a }}$ Constructed by application of the Deyo adaption [29-31] of the Charlson comorbidity index [32]

b Chi square

\section{a All stages}

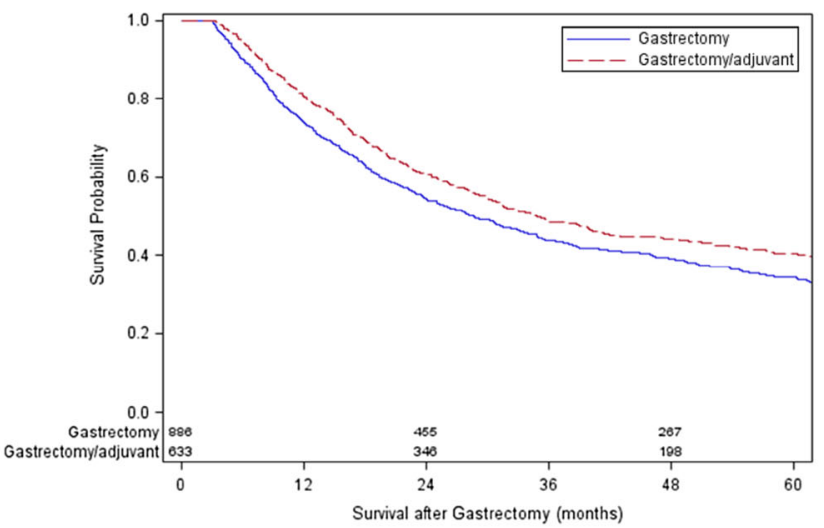

c Stage II

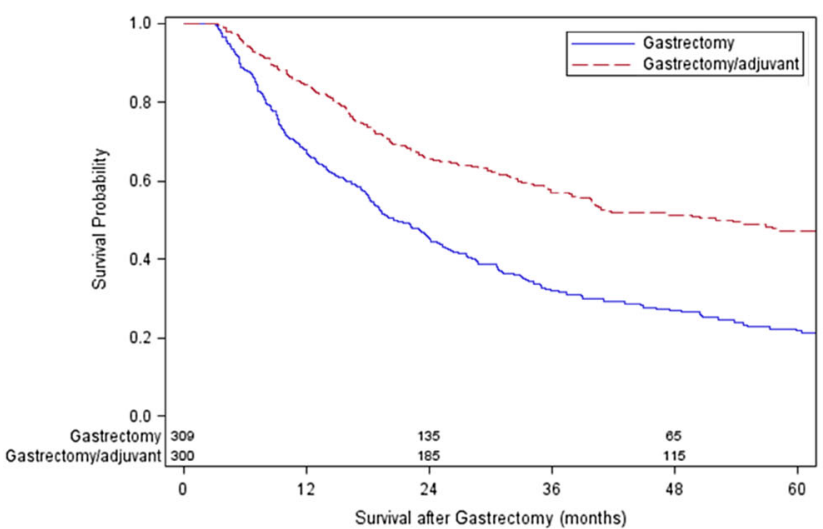

b Stage IB

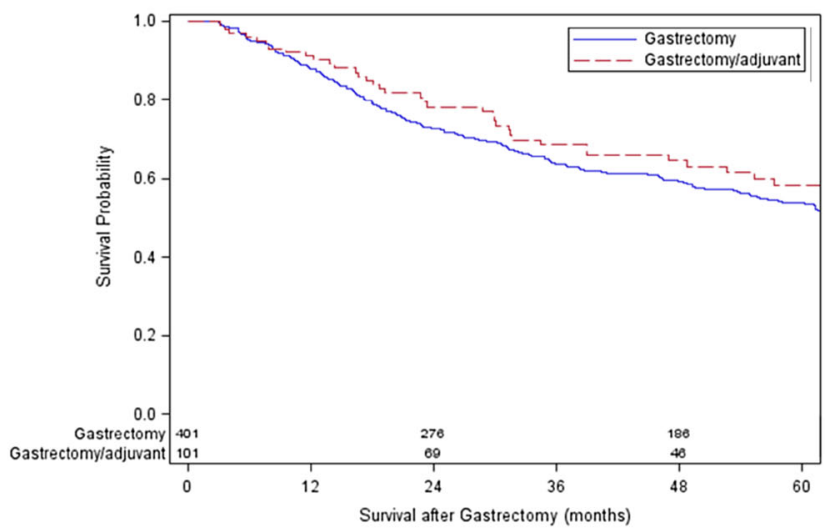

d Stage III

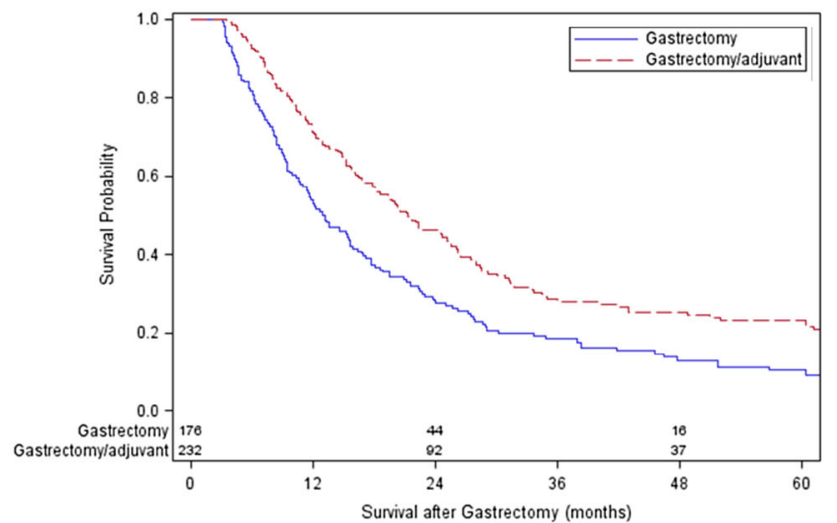

Fig. 2 Kaplan-Meier survival curves illustrating the survival of Medicare beneficiaries with diagnosed gastric adenocarcinoma according to the treatment regimen. a All patients, b patients with stage IB disease, $\mathbf{c}$ patients with stage II disease, and d patients with stage III disease. Number of individuals at risk at 12,36 , and 60 months masked 
Table 2 Crude median survival among patients in the two treatment groups and hazard ratios for overall survival after adjustment for patient characteristics

\begin{tabular}{|c|c|c|c|}
\hline \multirow[t]{2}{*}{ Characteristics } & \multicolumn{2}{|c|}{ Crude median survival from gastrectomy (months) ${ }^{\mathrm{d}}$} & \multirow{2}{*}{$\begin{array}{l}\text { Hazard ratio }^{\mathrm{e}} \\
\text { Gastrectomy only vs gastrectomy } \\
\text { plus adjuvant CRT }\end{array}$} \\
\hline & Gastrectomy only $(n=886)$ & Gastrectomy plus adjuvant CRT $(n=633)$ & \\
\hline All & $25.1(43.7)$ & $26.9(40.9)$ & 1.0 (reference) \\
\hline Unadjusted model & & & $0.83(0.73-0.95)^{\mathrm{f}}$ \\
\hline Adjusted model $^{\mathrm{a}}$ & & & $0.58(0.50-0.67)^{\mathrm{f}}$ \\
\hline \multicolumn{4}{|l|}{ Age at diagnosis (years) } \\
\hline $65-69$ & $28.1(45.7)$ & $29.9(49.2)$ & 1.0 (reference) \\
\hline 70-74 & $23.6(43.1)$ & $24.4(39.8)$ & $1.32(1.12-1.56)^{\mathrm{f}}$ \\
\hline $75-79$ & $25.0(42.6)$ & $28.3(37.5)$ & $1.23(1.03-1.47)^{\mathrm{f}}$ \\
\hline \multicolumn{4}{|l|}{ Sex } \\
\hline Male & $25.8(43.2)$ & $26.0(42.6)$ & 1.0 (reference) \\
\hline Female & $23.6(44.0)$ & $28.1(40.2)$ & $0.999(0.87-1.15)$ \\
\hline \multicolumn{4}{|l|}{ Race/ethnicity } \\
\hline Non-Hispanic white & $25.0(46.0)$ & $26.5(43.4)$ & 1.0 (reference) \\
\hline Non-Hispanic black & $17.7(35.9)$ & $28.3(40.5)$ & $1.07(0.87-1.32)$ \\
\hline Non-Hispanic Asian/other & $29.5(43.7)$ & $26.3(35.5)$ & $0.97(0.78-1.21)$ \\
\hline Hispanic & $29.0(45.7)$ & $30.7(44.5)$ & $0.97(0.77-1.21)$ \\
\hline \multicolumn{4}{|l|}{ Median income } \\
\hline 0 (lowest) & $22.4(44.8)$ & $28.0(38.4)$ & 1.0 (reference) \\
\hline 1 & $22.7(44.0)$ & $26.6(38.4)$ & $1.01(0.81-1.26)$ \\
\hline 2 & $23.7(40.1)$ & $23.4(38.7)$ & $1.08(0.86-1.35)$ \\
\hline 3 & $30.6(45.8)$ & $25.4(37.4)$ & $0.96(0.78-1.22)$ \\
\hline 4 (highest) & $26.0(46.5)$ & $30.8(50.2)$ & $0.91(0.70-1.19)$ \\
\hline \multicolumn{4}{|l|}{ College educated } \\
\hline 0 (lowest) & $22.3(37.1)$ & $25.2(40.0)$ & 1.0 (reference) \\
\hline 1 & $28.7(51.7)$ & $30.7(44.2)$ & $0.85(0.69-1.05)$ \\
\hline 2 & $22.8(44.1)$ & $24.1(34.7)$ & $0.90(0.72-1.12)$ \\
\hline 3 & $24.3(40.1)$ & $26.9(36.8)$ & $0.87(0.69-1.09)$ \\
\hline 4 (highest) & $31.2(47.3)$ & $26.9(52.3)$ & $0.85(0.65-1.10)$ \\
\hline \multicolumn{4}{|l|}{ SEER region } \\
\hline Northeast & $24.9(43.4)$ & $31.5(46.4)$ & 1.0 (reference) \\
\hline South & $23.0(44.7)$ & $24.7(40.3)$ & $1.13(0.92-1.38)$ \\
\hline Midwest & $25.1(38.8)$ & $24.5(34.6)$ & $1.17(0.91-1.51)$ \\
\hline West/Hawaii & $26.9(43.7)$ & $26.9(44.4)$ & $0.99(0.83-1.19)$ \\
\hline \multicolumn{4}{|l|}{ Birthplace } \\
\hline USA and US territories & $20.8(35.4)$ & $24.4(34.8)$ & 1.0 (reference) \\
\hline Foreign & $27.8(41.4)$ & $23.5(48.2)$ & $0.76(0.63-0.92)^{\mathrm{f}}$ \\
\hline Unknown & $38.9(57.9)$ & $32.6(49.9)$ & $0.41(0.34-0.48)^{\mathrm{f}}$ \\
\hline \multicolumn{4}{|l|}{ AJCC stage } \\
\hline IB & $44.2(51.1)$ & $39.3(47.0)$ & 1.0 (reference) \\
\hline II & $19.2(31.7)$ & $33.2(49.1)$ & $2.02(1.71-2.39)^{\mathrm{f}}$ \\
\hline III & $12.3(16.7)$ & $19.3(19.9)$ & $3.85(3.20-4.64)^{\mathrm{f}}$ \\
\hline \multicolumn{4}{|l|}{ Charlson comorbidity score ${ }^{\mathrm{b}}$} \\
\hline 0 & $28.6(44.6)$ & $29.1(46.9)$ & 1.0 (reference) \\
\hline 1 & $22.4(42.3)$ & $24.7(39.8)$ & $1.24(1.07-1.45)^{\mathrm{f}}$ \\
\hline $2+$ & $21.9(40.6)$ & $24.6(37.9)$ & $1.12(0.95-1.33)$ \\
\hline \multicolumn{4}{|l|}{ Tumor location } \\
\hline Cardia/overlapping & $20.5(38.0)$ & $24.9(37.7)$ & 1.0 (reference) \\
\hline
\end{tabular}


Table 2 continued

\begin{tabular}{|c|c|c|c|}
\hline \multirow[t]{2}{*}{ Characteristics } & \multicolumn{2}{|c|}{ Crude median survival from gastrectomy (months) ${ }^{\mathrm{d}}$} & \multirow{2}{*}{$\begin{array}{l}\text { Hazard ratio }^{\mathrm{e}} \\
\text { Gastrectomy only vs gastrectomy } \\
\text { plus adjuvant CRT }\end{array}$} \\
\hline & Gastrectomy only $(n=886)$ & Gastrectomy plus adjuvant CRT $(n=633)$ & \\
\hline Noncardia & $28.8(45.6)$ & $28.8(44.1)$ & $0.83(0.72-0.96)^{\mathrm{f}}$ \\
\hline \multicolumn{4}{|c|}{ Lauren classification } \\
\hline Intestinal & $26.9(45.2)$ & $29.2(41.8)$ & 1.0 (reference) \\
\hline Diffuse & $18.8(40.6)$ & $20.5(29.6)$ & $1.30(1.11-1.53)^{\mathrm{f}}$ \\
\hline Other & $23.9(39.0)$ & $26.9(48.8)$ & $1.12(0.89-1.41)$ \\
\hline \multicolumn{4}{|c|}{ Year of diagnosis } \\
\hline 2002-2004 & $28.9(65.8)$ & $33.9(67.8)$ & 1.0 (reference) \\
\hline 2005-2007 & 30.7 (38.6) & $34.4(35.4)$ & $0.95(0.82-1.10)$ \\
\hline $2008-2009^{c}$ & $16.4(14.8)$ & $18.8(13.2)$ & $1.01(0.81-1.25)$ \\
\hline \multicolumn{4}{|c|}{ Lymph nodes examined } \\
\hline$<15$ & $25.0(45.2)$ & $28.2(43.3)$ & 1.0 (reference) \\
\hline $15-25$ & $23.8(38.2)$ & $26.5(38.8)$ & $0.99(0.85-1.15)$ \\
\hline$>26$ & $28.7(43.5)$ & $22.6(38.9)$ & $0.84(0.68-1.03)$ \\
\hline Unknown & $19.9(22.3)$ & $15.6(57.6)$ & $1.16(0.72-1.86)$ \\
\hline
\end{tabular}

AJCC American Joint Committee on Cancer, CRT chemoradiotherapy, SEER Surveillance, Epidemiology, and End Results

${ }^{a}$ Adjusted model with adjustment for age at diagnosis, sex, race/ethnicity, marital status, college education, SEER region, birthplace, AJCC stage, Charlson comorbidity score, tumor location, Lauren tumor categorization, year of diagnosis, and number of lymph nodes examined

b Modified Charlson comorbidity score was constructed by application of the Deyo adaption [29-31] of the Charlson comorbidity index [32]

c Data available only until December 31, 2010. The 1-year postgastrectomy overall survival rate for gastrectomy only and adjuvant CRT was $74.6 \%$ versus $80.5 \%$ for $2002-2004,73.8 \%$ versus $81.9 \%$ for $2005-2007$, and $69.1 \%$ versus $77.1 \%$ for $2008-2009$

$\mathrm{d}$ The interquartile range is given in parentheses

e The $95 \%$ confidence interval is given in parentheses

${ }^{\mathrm{f}}$ Statistically significant $(p<0.05)$

\section{Results}

\section{Cohort description and patient characteristics}

From an initial sample of 25,099 patients in whom gastric adenocarcinoma was diagnosed between 2002 and 2009, we identified 2936 patients who met the inclusion criteria (see Fig. 1). Among these patients, 1924 underwent gastrectomy within 6 months of diagnosis, did not receive any neoadjuvant therapy (e.g., preoperative chemotherapy and/ or radiation therapy), and survived for more than 90 days after the procedure. A subset of 405 patients who received only adjuvant chemotherapy or radiation therapy within 3 months of gastrectomy were also excluded for the main analysis (but were included in sensitivity analysis), for a final cohort of 1519 patients. With adjuvant treatment defined as receipt of chemotherapy and radiation therapy within 3 months of gastrectomy, the final cohort included 886 patients $(58.3 \%)$ who underwent gastrectomy only and 633 patients $(41.7 \%)$ who underwent gastrectomy and received adjuvant CRT.

Compared with the gastrectomy-only group, adjuvant CRT treatment group had a higher proportion of patients younger than 75 years $(75.5 \%$ vs $55.3 \% ; p<0.0001)$. They were also likelier to be in the higher median income quintile $(28.4 \%$ vs $19.2 \% ; p=0.0002)$ and to have stage II or stage III cancer at diagnosis $(84.0 \%$ vs $54.7 \%$; $p<0.0001$ ) (Table 1).

Patients who were treated with gastrectomy only were likelier to have stage IB cancer at diagnosis $(45.3 \%$ vs $16.0 \% ; p<0.0001)$ or to have a Deyo modified Charlson comorbidity score of 2 or greater $(23.6 \%$ vs $19.0 \%$; $p=0.07$ ). Differences in baseline characteristics of race/ ethnicity, SEER region and birthplace were not statistically significant between the two groups (Table 1).

\section{Survival outcomes}

\section{Overall survival}

The median overall survival was 26.9 months [interquartile range (IQR) 40.9 months] for patients who also received adjuvant CRT within 3 months of gastrectomy versus 25.1 months (IQR 43.7 months) for patients who underwent gastrectomy only (Table 2). The Kaplan-Meier curves for overall survival are shown in Fig. 2. A greater 
Table 3 Estimated cancer-specific survival outcomes by treatment regimen

\begin{tabular}{|c|c|c|c|c|c|c|c|c|}
\hline \multirow{2}{*}{$\begin{array}{l}\text { Time after } \\
\text { gastrectomy } \\
\text { (months) }\end{array}$} & \multicolumn{2}{|l|}{ All stages } & \multicolumn{2}{|l|}{ Stage IB } & \multicolumn{2}{|l|}{ Stage II } & \multicolumn{2}{|l|}{ Stage III } \\
\hline & $\begin{array}{l}\text { Gastrectomy } \\
\text { only }(\%)\end{array}$ & $\begin{array}{l}\text { Gastrectomy } \\
\text { plus adjuvant } \\
\text { CRT }(\%)\end{array}$ & $\begin{array}{l}\text { Gastrectomy } \\
\text { only }(\%)\end{array}$ & $\begin{array}{l}\text { Gastrectomy } \\
\text { plus adjuvant } \\
\text { CRT }(\%)\end{array}$ & $\begin{array}{l}\text { Gastrectomy } \\
\text { only }(\%)\end{array}$ & $\begin{array}{l}\text { Gastrectomy } \\
\text { plus adjuvant } \\
\text { CRT }(\%)\end{array}$ & $\begin{array}{l}\text { Gastrectomy } \\
\text { only }(\%)\end{array}$ & $\begin{array}{l}\text { Gastrectomy } \\
\text { plus adjuvant } \\
\text { CRT }(\%)\end{array}$ \\
\hline 12 & 80 & 84 & 92 & 93 & 72 & 87 & 61 & 75 \\
\hline 36 & 54 & 56 & 75 & 73 & 41 & 66 & 24 & 35 \\
\hline 60 & 47 & 50 & 69 & 71 & 33 & 58 & 16 & 29 \\
\hline
\end{tabular}

$C R T$ chemoradiotherapy

proportion of patients who received adjuvant CRT were alive at 1 year after gastrectomy compared with those who underwent gastrectomy only ( $80.3 \%$ vs $73.5 \%$ ). Cancerspecific survival was similar between the two groups $(50 \%$ vs $47 \%$; see Table 3 ).

In an unadjusted Cox proportional hazards model, there was a significant difference in overall survival between the two treatment groups (hazard ratio $0.83 ; 95 \%$ CI 0.73-0.95) (Table 4). Controlling for baseline characteristics, the multivariable model also found a statistically significant overall survival benefit associated with gastrectomy plus adjuvant CRT (hazard ratio $0.58 ; 95 \%$ CI 0.50-0.67). All four propensity-score-adjusted models found a similar adjuvant CRT benefit. For example, the propensity-score-stratified model found that those who received adjuvant CRT in addition to gastrectomy had a $39 \%$ relative reduction in mortality (hazard ratio $0.61 ; 95 \%$ CI 0.41-0.79) compared with those who underwent only gastrectomy (Table 4).

\section{Stage-specific survival}

For patients with stage IB tumors, the median overall survival from the time of gastrectomy was similar between gastrectomy plus adjuvant CRT and gastrectomy alone [39.3 months (IQR 47.0) and 44.2 months (IQR 51.1)] (Table 2). In contrast, the addition of adjuvant CRT was associated with greater survival for patients with stage II [33.2 months (IQR 49.1) vs 19.2 months (IQR 31.7)] and stage III [19.3 months (IQR 19.9) vs 12.3 months (IQR 16.7)] cancers. For all stages at diagnosis, adjuvant CRT had a greater proportion of patients alive at 1 year after gastrectomy (stage IB $90.1 \%$ vs $87.3 \%$; stage II $84.0 \%$ vs $67.3 \%$; stage III $71.1 \%$ vs $52.8 \%$ ); the absolute difference was greater for more advanced tumors. Figure 2 depicts Kaplan-Meier survival curves by the stage at diagnosis. For cancer-specific survival at 1-year after gastrectomy, a greater proportion of stage II cancer ( $87 \%$ vs $72 \%$ ) and stage III cancer ( $75 \%$ vs $61 \%$ ) patients treated with the addition of adjuvant CRT were also alive compared with those who underwent gastrectomy only; outcomes among the two groups were similar however for stage IB cancer patients (93\% vs 92\%) (Table 3).

For stage IB tumors, adjusted multivariable models did not detect a survival difference between gastrectomy only and gastrectomy plus adjuvant therapy (hazard ratio 1.02; 95\% CI 0.71-1.45; Table 4). However, adjuvant CRT was associated with a statistically significant survival benefit for stage II (hazard ratio 0.50 ; 95\% CI $0.39-0.61$ ) and stage III (hazard ratio 0.58 ; $95 \%$ CI $0.45-0.73$ ) cancers. The results for propensity-score-adjusted models were similar (see Table 4).

\section{Subgroup analysis}

The survival benefit did not differ by tumor location or age at cancer diagnosis (Table 4). However, for all subgroup analyses, no significant survival benefit was detected for stage IB cancers.

\section{Sensitivity analysis}

The results were insensitive to assumptions on the adjuvant CRT treatment window [hazard ratio $0.63 \quad(95 \%$ CI 0.54-0.74) for 2 months and hazard ratio 0.59 (95\% CI 0.51-0.68) for 4 months]. Similarly, if adjuvant therapy was defined as any chemotherapy within 3 months (with or without radiation therapy) or only adjuvant chemotherapy, the treatment effects were largely unchanged [hazard ratio 0.63 (95\% CI $0.55-0.72)$ and hazard ratio 0.61 (95\% CI 0.52-0.70) respectively]. In contrast, if adjuvant therapy consisted of only radiation therapy (i.e., no chemotherapy) within 3 months of gastrectomy, no survival benefit was detected compared with gastrectomy only [hazard ratio $1.01 ; 95 \%$ CI $0.80-1.27)$. The results were consistent for overall and stage-specific survival (see Table 4).

\section{Discussion}

Using SEER-Medicare data since 2002 when adjuvant CRT became part of US standard care [4,5], we found that Medicare enrollees in whom gastric adenocarcinoma was 


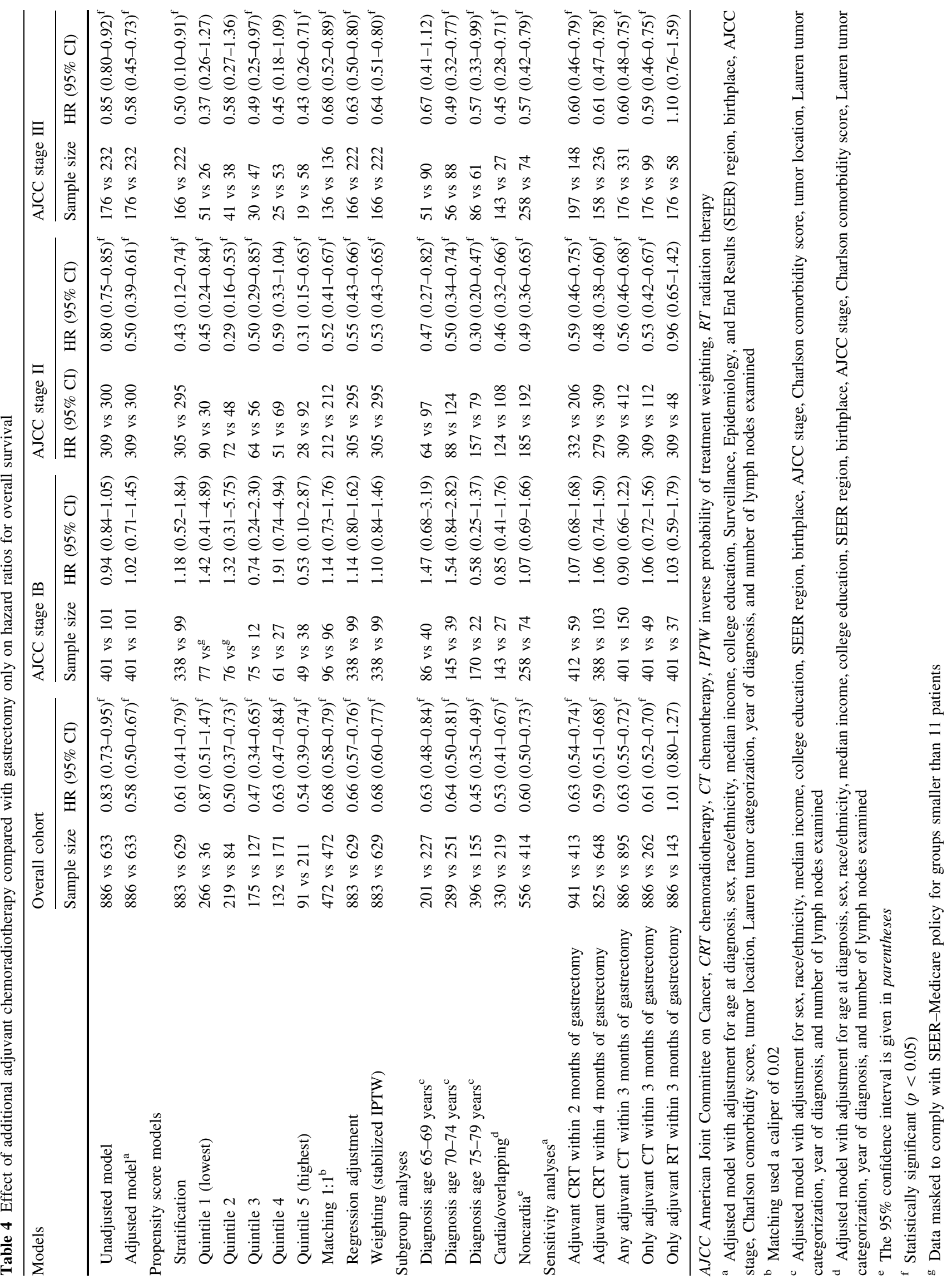




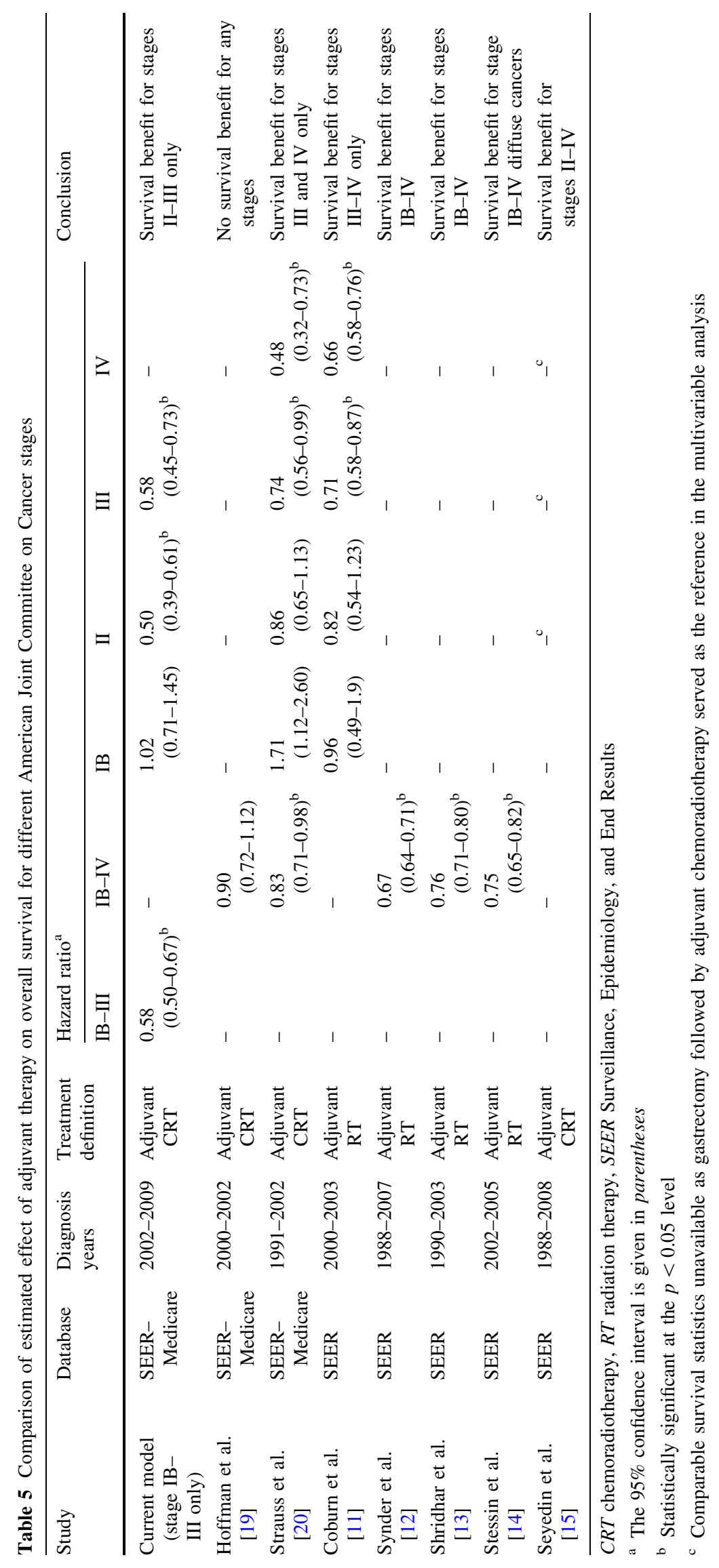


diagnosed between age 65 years and age 79 years who received adjuvant CRT in addition to gastrectomy had more favorable survival. These findings suggest that older patients, who account for the most of gastric cancer cases, benefit from adjuvant CRT. In particular, patients in whom a stage II or stage III nonmetastatic gastric adenocarcinoma had been diagnosed received an estimated $42-50 \%$ relative reduction in death risk. Patients in whom stage IB tumors had been diagnosed demonstrated a negligible survival benefit associated with adjuvant CRT.

Among our study cohort of older patients in whom gastric adenocarcinoma had been diagnosed who underwent gastrectomy, we found that only $42 \%$ received adjuvant CRT within 3 months of gastrectomy. The vast majority of these patients had stage II or stage III disease at diagnosis. The reasons for these low rates of postoperative adjuvant treatment are uncertain, and may include the emergence of neoadjuvant therapies. Of note, although our study cohort excluded patients who received neoadjuvant therapy, when we included these individuals in our cohort, we found that $11 \%$ of individuals who underwent gastrectomy $(n=2458)$ received some type of neoadjuvant therapy, with the proportion increasing over time from $5.8 \%$ in $2002-2004$ to $20.5 \%$ in $2008-2009$ (Table S2).

Prior studies used the SEER [11-15] and SEER-Medicare data $[19,20]$ to evaluate the association between adjuvant treatment and survival, primarily using data collected before CRT became part of standard care in 2002 or incomplete chemotherapy information. Using SEERMedicare data collected since 2002 that include detailed data on chemotherapy use, our current study adds to the growing body of evidence that suggests that adjuvant CRT, compared with gastrectomy alone, is associated with a survival benefit, in particular for patients with more advanced stage II and stage III tumors (Table 5).

With the ability to distinguish between different types of adjuvant therapy on the basis of chemotherapy information available in the SEER-Medicare database we were also able to assess the benefit associated with alternative regimens, including a statistically significant survival benefit for any adjuvant chemotherapy, regardless of receipt of radiation therapy, and a negligible benefit for adjuvant radiation therapy if it is administered without any chemotherapy. Although not directly comparable, our results are consistent with findings from the Adjuvant Chemoradiation Therapy in Stomach Cancer (ARTIST) trial, which found that the addition of radiation therapy to chemotherapy did not significantly reduce recurrence after curative resection and D2 lymph node dissection among patients with gastric cancer [41]. Similarly, the CRITICS study did not find any differences in 5-year survival rates among patients who received postgastrectomy chemotherapy and CRT (after neoadjuvant therapy) [42], suggesting little benefit from intensifying postoperative treatment with radiation therapy.

As with any observational study based on administrative data, our study is subject to limitations. The patient cohort was limited to those who were Medicare beneficiaries living in a SEER region and aged 65 years or older in 2002-2009. These patients are not representative of all US gastric cancer patients who underwent gastrectomy. Although the SEER-Medicare database is large, gastric cancer is uncommon in the USA. Therefore, our cohort size is too small to detect differences between certain subgroups. The database also does not have information on total radiation therapy dose, and it is possible that some patients in the adjuvant CRT group did not complete the course of treatment or received nonstandard doses. In addition, the SEER-Medicare database does not have complete information for patients who received care through an HMO. Some studies have suggested that patterns of care and comorbidities differ between Medicare patients and HMO patients [43, 44]. Although agreement between SEER and Medicare databases for surgical procedures was not completely concurrent, studies have shown high concordance between the two databases [45]. Since we anchored survival on the date of gastrectomy, approximately 300 patients listed as having surgery in the SEER database (with no information on the date of procedure) but not in the Medicare database were excluded. Lastly, although we used propensity score adjustment to account for imbalances between the two groups based on known factors, selection bias based on other unknown, unmeasured variables, such as tumor residual information, may have influenced treatment choice.

\section{Conclusion}

Our findings suggest that adjuvant CRT in conjunction with gastrectomy is associated with a survival benefit among older patients in whom stage II and stage III gastric adenocarcinoma has been diagnosed. Comparative effectiveness studies that capture both the morbidity risks and survival benefits associated with adjuvant CRT are needed to further identify opportunities to improve outcomes of gastric cancer treatment.

Acknowledgements Jennifer M. Yeh was supported by the National Cancer Institute of the National Institutes of Health (K07CA143044). This study used the linked Surveillance, Epidemiology, and End Results (SEER)-Medicare database. The interpretation and reporting of these data are the sole responsibility of the authors. The authors acknowledge the efforts of the National Cancer Institute; the Office of Research, Development, and Information, Centers for Medicare \& Medicaid Services; Information Management Services, Inc.; and the SEER Program tumor registries in the creation of the SEER-Medicare database. 


\section{Compliance with ethical standards}

Conflict of interest The authors declare that they have no conflict of interest.

Research involving human participants and/or animals This study does not contain any studies with human or animal subjects performed by any of the authors.

Informed consent This study does not contain any studies with human or animal subjects performed by any of the authors.

\section{References}

1. Ferlay J, Soerjomataram I, Dikshit R, Eser S, Mathers C, Rebelo $\mathrm{M}$, et al. Cancer incidence and mortality worldwide: sources, methods and major patterns in GLOBOCAN 2012. Int J Cancer. 2015;136(5):E359-86.

2. National Cancer Institute. Surveillance, Epidemiology, and End Results Program SEER*Stat Database: Incidence-SEER 9 Regs research data, Nov 2010 Sub (1973-2008) (Katrina/Rita population adjustment)—linked to county attributes-total U.S., 1969-2009 counties, National Cancer Institute, DCCPS, Surveillance Research Program, Cancer Statistics Branch, released April 2011, based on the November 2010 submission.

3. Ashraf N, Hoffe S, Kim R. Adjuvant treatment for gastric cancer: chemotherapy versus radiation. Oncologist. 2013;18(9):1013-21.

4. Macdonald JS, Smalley SR, Benedetti J, Hundahl SA, Estes NC, Stemmermann GN, et al. Chemoradiotherapy after surgery compared with surgery alone for adenocarcinoma of the stomach or gastroesophageal junction. $\mathrm{N}$ Engl $\mathrm{J}$ Med. 2001;345(10):725-30.

5. Smalley SR, Benedetti JK, Haller DG, Hundahl SA, Estes NC, Ajani JA, et al. Updated analysis of SWOG-directed intergroup study 0116: a phase III trial of adjuvant radiochemotherapy versus observation after curative gastric cancer resection. J Clin Oncol. 2012;30(19):2327-33.

6. Cunningham D, Allum WH, Stenning SP, Thompson JN, Van de Velde CJ, Nicolson M, et al. Perioperative chemotherapy versus surgery alone for resectable gastroesophageal cancer. N Engl J Med. 2006;355(1):11-20.

7. Ajani JA, Bentrem DJ, Besh S, D'Amico TA, Das P, Denlinger C, et al. Gastric cancer, version 2.2013: featured updates to the NCCN guidelines. J Natl Compr Cancer Netw. 2013;11(5):531-46.

8. Knight G, Earle CC, Cosby R, Coburn N, Youssef Y, Malthaner $\mathrm{R}$, et al. Neoadjuvant or adjuvant therapy for resectable gastric cancer: a systematic review and practice guideline for North America. Gastric Cancer. 2013;16(1):28-40.

9. Okines A, Verheij M, Allum W, Cunningham D, Cervantes A, Arnold D. Gastric cancer: ESMO clinical practice guidelines for diagnosis, treatment and follow-up. Ann Oncol. 2010;21(Suppl 5):v50-4.

10. Snyder RA, Penson DF, Ni S, Koyama T, Merchant NB. Trends in the use of evidence-based therapy for resectable gastric cancer. J Surg Oncol. 2014;110(3):285-90.

11. Coburn NG, Govindarajan A, Law CH, Guller U, Kiss A, Ringash J, et al. Stage-specific effect of adjuvant therapy following gastric cancer resection: a population-based analysis of 4,041 patients. Ann Surg Oncol. 2008;15(2):500-7.

12. Snyder RA, Castaldo ET, Bailey CE, Phillips SE, Chakravarthy AB, Merchant NB. Survival benefit of adjuvant radiation therapy for gastric cancer following gastrectomy and extended lymphadenectomy. Int J Surg Oncol. 2012;2012:307670.

13. Shridhar R, Dombi GW, Weber J, Hoffe SE, Meredith K, Konski A. Adjuvant radiation therapy increases overall survival in nodepositive gastric cancer patients with aggressive surgical resection and lymph node dissection: a SEER database analysis. Am J Clin Oncol. 2012;35(3):216-21.

14. Stessin AM, Sison C, Schwartz A, Ng J, Chao CK, Li B. Does adjuvant radiotherapy benefit patients with diffuse-type gastric cancer? Results from the Surveillance, Epidemiology, and End Results database. Cancer. 2014;120(22):3562-8.

15. Seyedin S, Wang PC, Zhang Q, Lee P. Benefit of adjuvant chemoradiotherapy for gastric adenocarcinoma: a SEER population analysis. Gastrointest Cancer Res. 2014;7(3-4):82-90.

16. Lordick F, Terashima M. Gastric cancer adjuvant therapy. Best Pract Res Clin Gastroenterol. 2016;30(4):581-91.

17. Dikken JL, Jansen EP, Cats A, Bakker B, Hartgrink HH, Kranenbarg EM, et al. Impact of the extent of surgery and postoperative chemoradiotherapy on recurrence patterns in gastric cancer. J Clin Oncol. 2010;28(14):2430-6.

18. Lordick F, Siewert JR. Recent advances in multimodal treatment for gastric cancer: a review. Gastric Cancer. 2005;8(2):78-85.

19. Hoffman KE, Neville BA, Mamon HJ, Kachnic LA, Katz MS, Earle CC, et al. Adjuvant therapy for elderly patients with resected gastric adenocarcinoma: population-based practices and treatment effectiveness. Cancer. 2012;118(1):248-57.

20. Strauss J, Hershman DL, Buono D, McBride R, Clark-Garvey S, Woodhouse SA, et al. Use of adjuvant 5-fluorouracil and radiation therapy after gastric cancer resection among the elderly and impact on survival. Int J Radiat Oncol Biol Phys. 2010;76(5):1404-12.

21. Warren JL, Klabunde CN, Schrag D, Bach PB, Riley GF. Overview of the SEER-Medicare data: content, research applications, and generalizability to the United States elderly population. Med Care. 2002;40(8 Suppl):IV-3-18.

22. National Cancer Institute. Surveillance, Epidemiology, and End Results Program. http://seer.cancer.gov (2014). Accessed 17 Jul 2014.

23. Engels EA, Pfeiffer RM, Ricker W, Wheeler W, Parsons R, Warren JL. Use of Surveillance, Epidemiology, and End ResultsMedicare data to conduct case-control studies of cancer among the US elderly. Am J Epidemiol. 2011;174(7):860-70.

24. Anderson JR, Cain KC, Gelber RD. Analysis of survival by tumor response. J Clin Oncol. 1983;1(11):710-9.

25. Greene FL, Page DL, Fleming ID, Fritz A, Balch CM, Haller DG, Morrow M, editors. AJCC cancer staging manual. 6th edn. New York: Springer; 2002.

26. Lauren PA. The two histological main types of gastric carcinoma: diffuse and so-called intestinal type carcinoma. Acta Path Microbiol Scand. 1965;64:31.

27. Henson DE, Dittus C, Younes M, Nguyen H, Albores-Saavedra J. Differential trends in the intestinal and diffuse types of gastric carcinoma in the United States, 1973-2000: increase in the signet ring cell type. Arch Pathol Lab Med. 2004;128(7):765-70.

28. Wu H, Rusiecki JA, Zhu K, Potter J, Devesa SS. Stomach carcinoma incidence patterns in the United States by histologic type and anatomic site. Cancer Epidemiol Biomarkers Prev. 2009;18(7):1945-52.

29. Deyo RA, Cherkin DC, Ciol MA. Adapting a clinical comorbidity index for use with ICD-9-CM administrative databases. J Clin Epidemiol. 1992;45(6):613-9.

30. Klabunde CN, Potosky AL, Legler JM, Warren JL. Development of a comorbidity index using physician claims data. J Clin Epidemiol. 2000;53(12):1258-67.

31. Romano PS, Roos LL, Jollis JG. Adapting a clinical comorbidity index for use with ICD-9-CM administrative data: differing 
perspectives. J Clin Epidemiol. 1993;46(10):1075-9; discussion 1081-90.

32. Charlson ME, Pompei P, Ales KL, MacKenzie CR. A new method of classifying prognostic comorbidity in longitudinal studies: development and validation. J Chronic Dis. 1987;40(5):373-83.

33. Rosenbaum PR, Rubin DB. The central role of the propensity score in observational studies for causal effects. Biometrika. 1983;70(1):41-55.

34. Rosenbaum PR, Rubin DB. Reducing bias in observational studies using subclassification on the propensity score. J Am Stat Assoc. 1984;79:516-24.

35. D'Agostino RB Jr, D'Agostino RB Sr. Estimating treatment effects using observational data. JAMA. 2007;297(3):314-6.

36. D'Agostino RB Jr. Propensity score methods for bias reduction in the comparison of a treatment to a non-randomized control group. Stat Med. 1998;17(19):2265-81.

37. Rubin DB. Estimating causal effects from large data sets using propensity scores. Ann Intern Med. 1997;127(8 Pt 2):757-63.

38. Austin PC. Optimal caliper widths for propensity-score matching when estimating differences in means and differences in proportions in observational studies. Pharm Stat. 2011;10(2):150-61.

39. Lunceford JK, Davidian M. Stratification and weighting via the propensity score in estimation of causal treatment effects: a comparative study. Stat Med. 2004;23(19):2937-60.
40. Robins JM, Hernan MA, Brumback B. Marginal structural models and causal inference in epidemiology. Epidemiology. 2000;11(5):550-60.

41. Lee J, Lim DH, Kim S, Park SH, Park JO, Park YS, et al. Phase III trial comparing capecitabine plus cisplatin versus capecitabine plus cisplatin with concurrent capecitabine radiotherapy in completely resected gastric cancer with D2 lymph node dissection: the ARTIST trial. J Clin Oncol. 2012;30(3):268-73.

42. Verheij M, Jansen EP, Cats A, van Grieken NC, Aaronson N, Boot $\mathrm{H}$, et al. A multicenter randomized phase III trial of neoadjuvant chemotherapy followed by surgery and chemotherapy or by surgery and chemoradiotherapy in resectable gastric cancer: first results from the CRITICS study. J Clin Oncol. 2016;34(Suppl):4000.

43. Riley G, Tudor C, Chiang YP, Ingber M. Health status of Medicare enrollees in HMOs and fee-for-service in 1994. Health Care Financ Rev. 1996;17(4):65-76.

44. Riley GF, Potosky AL, Klabunde CN, Warren JL, Ballard-Barbash R. Stage at diagnosis and treatment patterns among older women with breast cancer: an HMO and fee-for-service comparison. JAMA. 1999;281(8):720-6.

45. Cooper GS, Virnig B, Klabunde CN, Schussler N, Freeman J, Warren JL. Use of SEER-Medicare data for measuring cancer surgery. Med Care. 2002;40(8 Suppl):IV-43-8. 